\title{
The Burden of Influenza among Kenyan Pregnant and postpartum Women and their Infants, 2015-2020
}

\author{
Nancy Otieno ${ }^{1}$, Bryan Nyawanda ${ }^{1}$, Meredith McMorrow ${ }^{2}$, Martina Oneko ${ }^{1}$, Daniel \\ Omollo $^{1}$, Shirley Lidechi ${ }^{1}$, Marc-Alain Widdowson ${ }^{2}$, Brendan Flannery ${ }^{2}$, Sandra Chaves $^{2}$, \\ Eduardo Azziz-Baumgartner ${ }^{2}$, and Gideon Emukule ${ }^{2}$ \\ ${ }^{1}$ Kenya Medical Research Institute \\ ${ }^{2}$ Centers for Disease Control and Prevention
}

June 21, 2021

\begin{abstract}
Objective: To describe the burden of influenza among pregnant women and their young infants. Design: Prospective cohort study. Setting: Rural western Kenya. Population: Pregnant women below 31 weeks of gestation and their infants aged 6 months and below Methods: We conducted weekly follow-up until 6 months postpartum to identify acute respiratory illnesses (ARI). We collected nasal/nasopharyngeal and oropharyngeal swabs from mothers/infants with ARI and tested for influenza A and $\mathrm{B}$ using polymerase chain reaction. We calculated incidence of laboratory-confirmed influenza per 1,000 person-months. Main outcome measure: Incidence of medically attended influenza illness among pregnant women and its impact on birth outcomes. Results: During June 2015-May 2020, we enrolled 3,026 pregnant women at a median gestational age of 16 weeks (interquartile range [IQR], 13, 18) and followed 2,550 infants. Incidence of laboratory-confirmed influenza during pregnancy (10.3 episodes per 1,000 person-months [95\% CI 8.6-11.8]) was 2-fold higher than in the postpartum period (4.0 [95\% CI 2.6-5.5]; p <0.01), and significantly higher among HIV-infected pregnant women (15.6 [95\% CI 11.0-20.6] vs. 9.1 [95\% CI 7.5-10.8]; p<0.01). Incidence among young infants was 4.4 (95\% CI 3.0-5.9) and similar among HIV-exposed and HIV-unexposed infants. Conclusion: Our findings suggest a substantial burden of influenza illnesses during pregnancy, with a higher burden among HIV-infected mothers. Kenyan authorities should consider the value of vaccinating pregnant women, especially if HIV-infected. Funding: This work was supported by funding [Grant number GH002133] from the U.S. CDC, through the Influenza Division. Keywords: Burden, influenza, pregnant women, infants
\end{abstract}

\section{Hosted file}

Main Document.docx available at https://authorea.com/users/420965/articles/527161-the-burdenof-influenza-among-kenyan-pregnant-and-postpartum-women-and-their-infants-2015-2020

\section{Hosted file}

Figures 1-2.docx available at https://authorea.com/users/420965/articles/527161-the-burdenof-influenza-among-kenyan-pregnant-and-postpartum-women-and-their-infants-2015-2020 